\title{
¿Existe siquiera la educación de personas adultas? Reflexiones sobre un concepto diverso
}

\author{
Emilio Lucio-Villegas
}

\begin{abstract}
Resumen
En el momento presente la educación de personas adultas está siendo objeto de un proceso creciente de homogeneización, tanto en sus metodologías, como en sus contenidos, como, sobre todo, en sus finalidades. En el presente artículo queremos reflexionar y valorar la diversidad de la educación de personas adultas como un elemento de resistencia frente a las políticas y las prácticas del aprendizaje a lo largo de la vida que han reducido a la persona a mero trabajador y consumidor y la educación a un proceso individual dirigido, de forma exclusiva, a la incorporación al mundo del trabajo.Vamos a señalar diversas definiciones, modelos, ámbitos y dimensiones de la educación de personas adultas. Nos detendremos brevemente en la propia diversidad de la investigación en este campo. Concluimos señalando como la educación de personas adultas debe continuar siendo una propuesta alternativa, de resistencia a las tendencias dominantes y que potencie la participación de las personas.
\end{abstract}

Palabras clave:

comunidad; dialogo; experiencia; investigación; participación. 


\title{
Does adult education even exist? Reflections on a diverse concept
}

\begin{abstract}
At present moment adult education is under a increasing process of homogenisation, either in methodologies, contents or, overall, its aims. In this paper we want to reflect and valorise the diversity of adult education as an element of resistance against the policies and practices of Lifelong Learning that reduces the person to a mere worker and consumer and education to an individual process addressed to the labour market. We will indicate diverse definitions, spaces and dimensions of adult education. We will also stress the diversity in terms of research. We will conclude emphasise how adult education must continue to be an alternative setting of resistance to the dominant tendencies and encouraging people's participation.
\end{abstract}

Key words: Community; dialogue; experience; research; participation

\section{Será que ainda existe educação de adultos? Reflexões sobre um conceito marcado pela diversidade}

Resumo: Na atualidade, a educação de adultos é objeto de um processo de crescente homogeneização, tanto em termos metodológicos, como de conteúdos, e, sobretudo, de finalidades. No presente artigo, pretendemos refletir e destacar a diversidade que caracteriza a educação de adultos enquanto elemento de resistência relativamente às políticas e às práticas de aprendizagem ao longo da vida que reduziram as pessoas a meros trabalhadores e consumidores, bem como a educação a um processo individual dirigido, de forma exclusiva, à inserção no mundo do trabalho. É nossa intenção assinalar diversas definições, modelos, âmbitos e dimensões da educação de adultos. Enfatizamos brevemente a diversidade da investigação neste campo. Concluímos assinalando que a educação de adultos deve manter-se uma proposta alternativa, de resistência a tendências dominantes, que potencie a participação dos sujeitos.

Palavras-chave: comunidade; diálogo; experiencia; investigação; participação

\section{L'éducation des adultes existe encore ? Réflexions à propos d'un concept marqué para la diversité}

Résumé: Actuellement, l'éducation des adultes est objet d'un procès d’homogénéisation, dans les méthodologies, les contenus et, surtout, les finalités. Dans cet article, nous voulons discuter et valoriser la diversité de l'éducation d'adultes en tant qu'élément de résistance aux politiques et aux pratiques d'apprentissage tout au long de la vie qui ont réduit la personne à un simple travailleur et a un consommateur, bien que l'éducation a un procès individue dirigé exclusivement à l'insertion au monde du travail. Nous signalons différentes définitions, modèles, approches et dimensions de l'éducation des adultes. Nous nous détenons brièvement à la diversité de la recherche dans ce champ. Nous concluons en remarquant que l'éducation des adultes doit se maintenir une proposition alternative, de résistance aux tendances dominantes et qui doit valoriser la participation des personnes.

Mots clés: communauté; dialogue; expérience; recherche; participation 


\section{Introducción}

La educación de personas adultas (EPA en adelante) es un concepto escurridizo y diverso debido a sus prácticas, a las diferentes tradiciones de las que deriva, a los escenarios donde se produce y a las personas que participan en ella. Además, el propio concepto ha sufrido profundas transformaciones - incluida la de su denominación, ahora se habla de aprendizaje a lo largo de la vida (ALV en adelante) - en los últimos 20 años. Encontramos, además, la aparición de otras tradiciones educativas en el escenario mundial - China y la República de Corea principalmente (Rubenson y Elfert, 2014) - o los intentos en Europa de construir una educación ligada a la llamada sociedad del conocimiento que se centra, básicamente, en dar respuesta a las necesidades del mundo del trabajo.

En el presente artículo queremos recuperar el concepto de EPA - diverso y disperso - no sólo a partir de la discusión sobre diversas definiciones, sino explorando sus espacios de actuación, dimensiones y ámbitos de investigación para recuperar la visión de una educación cuyo foco se encuentra en las personas y las comunidades.

\section{El concepto de educación de personas adultas}

Definir la EPA puede ser una tarea compleja. Se ha considerado un concepto difuso y escurridizo que contiene diversos significados y diferentes prácticas. Como señala McCullogh:

Extraer la educación de adultos [sic] del medio social que le rodea - o al menos diferenciar la educación de adultos de su medio social - es tan difícil como determinar cuántos ángeles pueden bailar en la cabeza de un alfiler ¿La educación de adultos es una práctica o un programa? ¿Una metodología o una organización? ¿Una "ciencia" o un sistema? ¿Un proceso o una profesión? ¿La educación de adultos es diferente de la educación continua, la educación vocacional, la educación superior? ¿La educación de adultos posee forma y sustancia, o simplemente llena el ambiente como el aire? ¿Está, por lo tanto, la educación de adultos en todas partes, pero en ningún sitio en particular? ¿Existe siquiera la educación de adultos? (En Jarvis, 1989, p. 23, comillas en el original).

Considerando estas dificultades en la definición, podemos buscar otras formas diferentes de definir la educación de personas adultas. En Aprender a ser, Faure et al. (1986, p. 289) señalan:

La educación de los adultos [sic] responde a múltiples definiciones: es el sustitutivo de la educación primaria para gran parte de los adultos [sic] en 
el mundo; es el complemento de la educación elemental o profesional para numerosos individuos que solo han recibido una enseñanza muy incompleta; prolonga la educación de aquellos a quienes ayuda a hacer frente a las exigencias nuevas de su medio ambiente; perfecciona la educación de quienes poseen una formación de alto nivel; constituye, en fin, un modo de expresión individual para todos. Algunos aspectos pueden ser más importantes que otros en tal o cual país, pero todos tienen su razón de ser por doquier. Ya no es posible ni limitar la educación de los adultos [sic] al nivel rudimentario, ni encerrarla en el marco de una educación "cultural" para uso de una minoría. (cursiva y comillas en el original).

En una dirección similar, Barbier (2009) habla de formation des adultes (formación de las personas adultas), y se pregunta a sí mismo como puede ser reconocida. En su búsqueda de una definición, describe las actividades que pueden ser consideradas como esta formation des adultes: trabajo social, asuntos relacionados con el medio ambiente, herramientas comunicativas, asuntos relativos al mundo del trabajo y sus espacios, terapias o vida espiritual (2009).

Esta diversidad, y la vaguedad en definir el concepto, es un elemento esencial para comprender la EPA. La diversidad es tanto en términos conceptuales como geográficos. De hecho, cuando Lima y Guimarães analizan las políticas y prácticas del ALV consideran que han roto "la heterogeneidad que es un rasgo característico de la educación de personas adultas en muchos países europeos" (2011, p. 105). Por otro lado, es importante destacar que esta diversidad parece ser una garantía cuando hablamos de una educación comprometida con las personas y las comunidades. Como señala Gelpi (2004, p.153):

La educación de personas adultas en Europa parece progresivamente olvidar su historia hecha de luchas, resistencias, creatividades y haberse convertido en un instrumento del poder, usado sólo para el desarrollo personal y en la lógica del mercado. La prioridad parece ser el desarrollo económico, la productividad, el refuerzo de la "ciudadanía" en toda su ambigüedad (pues la finalidad no declarada es a menudo la de evitar los conflictos sociales y no la de oponerse a sus causas) (cursiva, comillas y paréntesis en el original).

En cualquier caso, nos parece que no es posible definir la EPA sin hacer referencia a los documentos, publicaciones y trabajos de la UNESCO.

La expresión educación de adultos [sic] designa la totalidad de los procesos organizados de educación, sea cual sea el contenido, el nivel o el método, sean formales o no formales, ya sea que prolonguen o reemplacen la 
educación formal dispensada en las escuelas y universidades, y en forma de aprendizaje profesional, gracias a las cuales las personas consideradas como adultos [sic] por la sociedad a la que pertenecen, desarrollan sus aptitudes, enriquecen sus conocimientos, mejoran sus competencias técnicas o profesionales o les dan una nueva orientación, y hacen evolucionar sus actitudes o su comportamiento en la doble perspectiva de un enriquecimiento integral del hombre [sic] y una participación en un desarrollo socioeconómico y cultural equilibrado e independiente (1977, p. 124, cursiva en el original).

De esta amplia definición de la UNESCO podemos derivar una serie de elementos. En primer lugar la clara dimensión cultural de la EPA, que, en este caso, tiene que ver con la diversidad geográfica que enmarca sus diversos enfoques. Por otro, el amplio espectro de actividades que pueden ser consideradas EPA, y, sobre todo, las profundas conexiones entre el desarrollo personal y la vida comunitaria y social de las personas.

Se puede considerar que la EPA está caracterizada, también, en términos de metodología y de las personas participantes en el proceso de enseñanza/aprendizaje. Se trata, en nuestra opinión, de una metodología que mezcla la vida cotidiana de las personas - individual y social - con el currículo. Se puede decir que la EPA está relacionada con la posibilidad de leer y decir el mundo al mismo tiempo que las personas leen y dicen palabras. Las personas llegan a ser más conscientes de sus propias situaciones partiendo de las palabras y los temas generadores como fundamentos del proceso (Freire, 1985).

Finger y Asún (2001) siguen a Ivan Illich al considerar que la EPA puede caracterizarse por: i) aprendizaje como opuesto a escolarización, ii) convivencia como opuesta a manipulación, iii) responsabilización como opuesta a desresponsabilización, y iv) participación como opuesta a control. Más adelante nos detendremos en la participación porque "en educación de personas adultas el conocimiento es creado por las personas, no para las personas" (Finger y Asún, 2001, p. 13, cursiva en el original).

Estas cuestiones son muy importantes, además, en un momento en el que la EPA parece orientarse hacia la responsabilidad individual del aprendiente - no como un derecho de las personas - y como una inversión de la que extraer beneficios.

En otro lugar, hemos construido una definición de EPA relacionada con la vida cotidiana que las personas tienen en las comunidades, y en el proceso de construir relaciones con los demás.

Educación de personas adultas es aprender democracia, investigar en las comunidades, buscar una escuela para todos y todas, transformar las comunidades, la lucha por nuestros propios derechos, llegar a tener conciencia de los peligros medioambientales, edificar la ciudad o expresarnos nosotros 
mismos a través del teatro o el arte en espacios públicos. Estas son cosas que un verdadero proceso educativo y de participación popular debería valorizar. Los conceptos y prácticas del aprendizaje a lo largo de la vida parecen haber olvidado que la vida es más que el mundo del trabajo. La vida incluye: parientes, relaciones de pareja y con los/as amigos/as, padres e hijos, vacaciones y trabajo, deseos e ilusiones, felicidad y tristeza, buenas y malas decisiones, emociones y sentimientos, un atardecer en Almograve o un amanecer en un avión volviendo a casa, el sucio y delicado oficio de vivir. Los conceptos y las prácticas del aprendizaje a lo largo de la vida parecen haber olvidado que la vida de las mujeres y los hombres es la substancia de la que está hecha la educación de personas adultas (Lucio-Villegas, 2009, pp. xiii-xiv).

\section{Modelos de Educación de personas adultas}

Se han propuesto diferentes modelos de EPA en un intento para estructurar la diversidad de la misma. Generalmente, los diversos modelos están dirigidos a diferenciar entre uno basado en la escolarización y el currículo y otro que presta más atención a construir una respuesta a los problemas y deseos de las personas y que se encuentra más relacionado con un enfoque comunitario.

Como un ejemplo, Jarvis (1986, pp. 71-72) diferencia entre una educación liberal y otra que denomina educación radical de personas adultas. Lo que el autor plantea es la diferenciación entre una educación que inicia a las personas en el conocimiento aceptado socialmente y otra que intenta potenciar la construcción del conocimiento y la creación de juicios propios para que las personas puedan desarrollar su potencial.

Siguiendo a Freire (1985) podemos diferenciar entre educación bancaria y educación liberadora - o crítica. Como señalan Dale y Hyslop-Marginson (2002, p.82): "El concepto de educación bancaria en Freire es el más reconocible de todo su trabajo. El término ha sido discutido y distorsionado [...] incluso maestros/as que se consideran ellos/as mismos/as progresistas son probablemente culpables de educación bancaria durante algunos momentos de su enseñanza".

Para Freire (1985, p. 78), la educación bancaria es definida, básicamente, porque "el educador [sic] es siempre el que educa; el educando [sic], el que es educado [...] finalmente, el educador es el sujeto del proceso; los educandos meros objetos"

La educación liberadora está relacionada con los procesos de concientización y el camino para alcanzar la autonomía y la emancipación. Freire hablaba de humanización y consideraba que el proceso de educación liberadora es una praxis: la reflexión y la acción de los hombres y de las mujeres sobre su realidad circundante a fin de transformarla. En la educación liberadora, los educandos [sic] van desarrollando su poder de captación y comprensión del mundo que, en sus relaciones con él, se les presenta no 
ya como una realidad estática sino como una realidad en transformación, en proceso (Freire, 1985, p. 94).

Otros autores y autoras han introducido un nuevo enfoque al incorporar el papel de los movimientos sociales y también el modelo dominante en la actualidad, basado en la formación de recursos humanos, y no en la educación de las personas. En esta línea Lima y Guimarães (2011) diferencian tres tipos:

a) El modelo democrático y emancipador, relacionado con el concepto de emancipación y basado en el trabajo de los movimientos sociales, principalmente. Se trata de "reinventar la democracia y la participación, particularmente a través de los movimientos sociales" (p.43).

b) El modelo que denominan como modernización y control del estado, que se caracteriza por "subestimar la acción de organizaciones ligadas a la sociedad civil" (Lima y Guimarães, 2011, p. 49). Si el anterior se caracteriza por la importancia dada a las actividades educativas no formales y/o informales, éste se encuentra, principalmente, centrado en la escuela y la escolarización.

c) El que denominan de recursos humanos que se encuentra "centrado en la adquisición de herramientas (que no se encuentran fomentadas en la oferta disponible en la actualidad en el sistema educativo)" (Lima y Guimarães, 2011, p. 58, paréntesis en el original). El logro más impresionante de este modelo es que ha recontextualizado los discursos sobre participación y educación popular para adaptarlos a un modelo no liberador y sí explotador. Como señalan, "herramientas, cualificación y certificados han originado un modelo pedagógico que expresa un cambio en el dominio educativo" (Lima y Guimarães, 2011, p. 54).

A partir de los modelos propuestos hasta ahora, podemos considerar que los autores y autoras citadas mantienen un acuerdo general sobre la existencia de, al menos, dos enfoques generales. Un enfoque relacionado con la liberación y la construcción de autonomía por parte de las personas y otro más interesado en la preservación de las relaciones sociales hegemónicas. El modelo de formación para/en el trabajo propuesto por diversidad de autores puede, en nuestra opinión, encontrarse situado en cualquiera de los dos anteriores. Un aprendizaje para la productividad, o uno que permita a las personas comprender el proceso productivo, el mundo del trabajo y las culturas del trabajo (Gelpi, 2004).

\section{Ámbitos de la educación de personas adultas}

En su investigación sobre la situación de la educación en el mundo durante los años sesenta del pasado siglo, Coombs (1978) descubrió la existencia de una gran diversidad de prácticas educativas. Una de sus características principales era que muchas de ellas tenían lugar fuera de la escuela y del sistema escolar. Desde ahí, diferenció entre educación formal, no formal e informal. De acuerdo con Coombs, el elemento más sorprendente era la riqueza - en su financiación y en términos educativos - de las 
iniciativas y experiencias que tenían lugar fuera de la escuela. De esta forma, se puede considerar que las escuelas no son los únicos espacios que proveen de educación. De hecho, una EPA de base comunitaria organizaría los procesos educativos tomando como fundamento el lugar donde las personas viven.

El principal argumento para diferenciar entre formal, no formal e informal está basado en dos dimensiones: intención e institución (Trilla en Lucio-Villegas, 2016) Intención significa que los fines del proceso educativo están claramente definidos como fines educativos. Institución se refiere a que las instituciones responsables de los procesos son definidas como tales y gozan del estatus como agentes educativos reconocidos socialmente. En otro lugar (Lucio-Villegas, 2016), hemos indicado como este esquema puede ser muy útil, pero es conveniente repensarlo para analizar experiencias que, dentro de la escuela, pueden no ser consideradas estrictamente formales.

\section{Dimensiones de la Educación de personas adultas}

Presentamos a continuación una serie de dimensiones que deberían ayudarnos a caracterizar un determinado modelo de EPA. Estas son: diálogo, participación, un enfoque basado en lo colectivo, la construcción colectiva del conocimiento, experiencia, diversidad y autonomía y emancipación.

\section{Diálogo}

El diálogo es el centro de la propuesta metodológica y filosófica de Paulo Freire. E diálogo garantiza la comunicación y define la educación como un proceso cooperativo, caracterizado por las interacciones sociales entre las personas, en el que un nuevo conocimiento es creado a partir de compartir el que las personas ya poseen. El diálogo significa múltiples voces y múltiples direcciones. En este diálogo múltiple y diverso el conocimiento es construido al mismo tiempo que el propio proceso tiene lugar. En este sentido, es el punto de partida para construir la educación liberadora.

Freire (1985) indicaba que la enseñanza y el aprendizaje son dos pasos en el proceso de crear conocimiento. Destacaba que realizar un trabajo colaborativo significa incluir a los miembros de una comunidad y construir los procesos educativos partiendo de su vida cotidiana a través de los temas o las palabras generadoras que emergen en el transcurso de la codificación y descodificación. En el mismo, las personas acometen un trabajo colectivo basado en la cooperación y la experiencia. Es en este proceso cuando el conocimiento de las personas emerge, creando uno nuevo sobre su realidad circundante, permitiendo reflexionar para transformar su realidad - su comunidad, su barrio, su pueblo.

\section{Participación}

La educación de personas adultas debe ser una educación participativa y que potencie la participación. Como señalan Botkin, Elmandjara y Malitza (1979): "La 
participación es algo más que limitarse a compartir formalmente las decisiones: es una actitud caracterizada por la cooperación, el diálogo y la empatía" (p. 35). Por ello, la EPA debe ser participativa en el sentido de que las personas adultas se comprometen en ella cuando la relacionan con su vida laboral, personal y comunitaria.

Es importante destacar que, en términos de EPA la participación implica también la construcción colectiva del conocimiento desenvolviendo una reflexión y una experiencia mutua que sea educativa (Olesen, 1989). Más adelante desarrollaremos estas dos dimensiones.

Un enfoque basado en lo colectivo

Este enfoque presenta un doble sentido. Por un lado, nos gustaría detenernos brevemente en los enfoques psicológicos derivados de autores como Vygotski, Luria o Wertsch entre otros. Ellos han investigado sobre la importancia de las interacciones sociales que las personas realizan en su vida cotidiana, para construir su personalidad y ayudar a desarrollar lo que denominan los procesos psicológicos superiores. De acuerdo con estos autores, nuestras herramientas conceptuales, nuestra manera de producir pensamiento complejo, son primero sociales y luego individuales. Como Luria (1987) demostró en su investigación sobre los Uzbezkis, la edificación de nuestras posibilidades de pensar sobre el mundo están relacionadas con el contexto y las circunstancias de nuestra vida.

El segundo sentido al que nos referimos al hablar de un enfoque basado en lo colectivo tiene que ver con el concepto/noción de comunidad. A partir de una extensa revisión de la literatura, Fragoso (2009) considera el término comunidad desde una perspectiva histórica, como una construcción social llena de significados y sentidos para las personas, como un territorio geográfico, o como un mecanismo de cambio - o para investigar sobre el cambio en las sociedades.

Ware (1986) define la comunidad como un lugar que "provee el ambiente para la vida individual" (p. 7). En ese sentido, esta autora considera que se trata de algo más que un territorio. La comunidad es, también, una forma de relacionarse entre personas que comparten recursos, conocimientos, instituciones, tradiciones, etc. Kurantowicz (2008) establece una distinción entre comunidad entendida como un lugar - un territorio geográfico - y un espacio - un territorio simbólico. Lugar y espacio se unen cuando las personas tienen un sentido de pertenencia no sólo al lugar, sino a la cultura y a los elementos simbólicos que la comunidad posee.

\section{La construcción colectiva del conocimiento}

Existen diversas y diferentes fuentes para construir y desarrollar conocimiento, y esto ocurre en las comunidades donde las personas viven. Para Toulmin (1977) sólo podemos comprender los conceptos si comprendemos los procesos socioculturales que se construyen en los lugares donde las personas viven. Estos procesos de producción colectiva 
del conocimiento se encuentran presentes en todas las actividades relacionadas con la EPA. Es el rescate del llamado conocimiento tradicional o popular que es siempre compartido y colectivo. Este conocimiento tradicional puede ser definido como:

Literatura, arte o trabajos científicos fundamentados en la tradición; representaciones; invenciones; descubrimientos científicos; diseños; modelos, nombres y símbolos; informaciones no reveladas; y otras innovaciones y creaciones basadas en la tradición, resultado de la actividad intelectual en los campos industrial, científico, literario o artístico (World Intellectual Property Organization, en Schroeder, 2009, p. 37).

En esta dirección de la construcción colectiva del conocimiento se encuentran también los trabajos sobre investigación participativa desarrollados por Orefice (1987) en el sur de Italia. En esos procesos, dos conocimientos - uno popular producto de la experiencia y las vivencias de las personas y otro académico proveniente del mundo universitario - se comprometieron en un diálogo en el que uno y otro se complementaban mutuamente en un proceso de co-creación cuyo resultado es un nuevo conocimiento.

\section{Experiencia}

La experiencia es un elemento estratégico para construir una EPA plena de significación para las personas. La experiencia es, de alguna forma, el resultado - y el proceso - por el que una persona organiza su conocimiento y lo comparte con las otras. Desde una perspectiva freireana, la experiencia se encuentra relacionada con la educación problematizadora y es la fuente para organizar los procesos de enseñanza y aprendizaje en el sentido de que puede ser considerada el germen desde donde las codificaciones emergen.

Olesen (1989, p.105) señala:

Por otro lado, nosotros buscábamos asegurar que los cursos funcionasen como una ilustración práctica del hecho que es posible cambiar un curso hacía la producción de experiencia. Profundizamos para incluir y explotar la experiencia de los participantes, construir sobre ella y hacerla visible (cursiva en el original).

De alguna forma, la educación es experiencia en un doble sentido. Por un lado, porque una experiencia es una reflexión afectiva sobre los acontecimientos que las personas vivencian. Por otro, es importante desarrollar metodologías que promuevan oportunidades para construir esas experiencias y aprender de ellas (Olesen, 1989). De hecho, se podría afirmar que en EPA, las escuelas y otros espacios educativos son lugares donde las personas comparten sus experiencias. 


\section{Diversidad}

El asunto de la diversidad es posible contemplarlo desde dos puntos de vista. primero está relacionado con la diversidad de actividades que pueden ser consideradas EPA, como ya señalamos al hablar de los ámbitos anteriormente y se recogía, por ejemplo, en algunas de las definiciones presentadas más arriba (Faure et al, 1986, UNESCO, 1997). También podemos plantearla al hablar de los espacios donde la EPA tiene lugar: escuelas y universidades, movimientos sociales y asociaciones, universidades populares, empresas, etc. Pero el elemento esencial de la diversidad tiene que ver con la variedad de personas involucradas en estas actividades: mujeres, hombres, adultos y adultas mayores, inmigrantes, trabajadores y trabajadoras, y jóvenes. Para caracterizar a esta diversidad de personas podemos utilizar una variedad de criterios.

a) Edad. Es un elemento legal y cultural que cambia a través de la historia y de una sociedad a otra. ¿Quién es una persona adulta en una sociedad occidental y quién es considerado como persona adulta en una sociedad africana?

b) Un segundo criterio está relacionado con las necesidades y los deseos que las personas atesoran para volver a ser estudiantes. Podemos diferenciar, al menos: i) las personas que tienen necesidad de un diploma para acceder al mundo del trabajo, y ii) las personas que buscan la adquisición de nuevos conocimientos para el disfrute de su tiempo libre, para la creación cultural y la expresión individual y colectiva, etc.

c) El contexto en el cual se produce la educación de las personas adultas. Este elemento se encuentra muy relacionado con la experiencia vivida tanto en la escuela como en la vida personal y social. El contexto donde las personas viven nos parece fundamental y, por ello, tenemos muchas reservas sobre las transferencias de buenas prácticas tal y como son presentadas en algunos estudios y documentos de la Unión Europea.

\section{Autonomía y Emancipación}

Desde sus primeros trabajos Freire consideró la educación como un proceso de liberación que debía permitir a las personas salir de la Cultura del Silencio y tener la experiencia de - y la confianza para - decir su palabra. Para mantener a estas personas en su situación de opresión, siempre siguiendo a Freire (1985), los sectores dominantes de la sociedad mantienen un sistema educativo que Freire llamó educación bancaria, y al que nos hemos referido más arriba.

Para alcanzar la emancipación Freire (1985) utiliza la noción/proceso de concientización, que es uno de sus más controvertidos conceptos. De hecho, dejó de utilizarlo al considerar que estaba siendo entendido en un sentido epistemológico: un hombre o una mujer oprimidos pueden ser conscientes de su opresión sólo de forma intelectual. La concientización requiere de prácticas sociales para constituirse como un camino de emancipación para las personas y las comunidades. 
Freire diferenció entre diversos niveles de conciencia: mágica, ingenua y crítica, que es la que permite realizar análisis sobre la realidad contextual y transformarla. El paso desde una a otra supone el compromiso con prácticas sociales transformadoras. Lo que resulta importante de esta noción/proceso de concientización es que se construye en la forja de las acciones que las personas emprenden en su vida cotidiana para, más allá de ser conscientes de su alienación, cambiar la situación que la produce. El término emancipación nos parece más coherente con un abordaje colectivo dirigido a las personas que viven en comunidad, mientras que empoderamiento parece estar más relacionado con personas individuales o grupos específicos dentro de las comunidades (Inglis, 1997).

\section{Un breve apunte sobre la investigación en educación de personas adultas}

Aunque sus trabajos se centran en estudios bibliométricos, Fejes y Nylander (2015) nos ofrecen una panorámica del estado de la investigación en la actualidad, con algunas reservas que señalaremos más adelante.

Para estos autores se produce un cambio desde los años 70 del siglo pasado, con una gran preocupación epistemológica y sobre el propio estatus de la educación de personas adultas, hasta la actualidad en el que las preocupaciones investigadoras están más relacionadas con el campo de estudio y las prácticas llevadas a cabo en el mismo, y con un abordaje mucho más interdisciplinar, por lo que consideran el campo de la investigación mucho más pluralista.

Los temas de investigación, referidos a los Estados Unidos y los países del norte de Europa, se focalizan en el estudio de los programas y métodos de enseñanza/ aprendizaje, la eficacia de los sistemas educativos, el desarrollo de la ciudadanía, o cómo integrar la EPA dentro de las políticas sociales y económicas - esta última línea es, posiblemente, la dominante en este momento.

En esta línea de tradiciones de investigación ligadas a ámbitos geográficos concretos, Rubenson y Elfert (2014) también diferencian entre una tradición norteamericana y una europea, pero destacan la irrupción de China y la República de Corea que incluyen nuevas y diferentes perspectivas, y una mayor fragmentación y especialización en los campos de estudio.

Como ya hemos señalado, el análisis de Fejes y Nylander se basa en tres prestigiosas revistas en el ámbito anglosajón, y en una mirada a la investigación en los países del llamado norte de Europa. Las preocupaciones investigadoras en los países del sur han estado más ligadas a su propio contexto y se acercan mucho más a la investigación sobre alfabetización, las relaciones y el papel de la EPA en el desarrollo de la comunidad, incluyendo la influencia de las transformaciones sociales en la vida cotidiana de las personas, o la presencia de estudiantes universitarios adultos y adultas y las limitaciones institucionales.

En relación a los métodos utilizados en las investigaciones, el estudio de Käpplinger (2015) concluye en la hegemónica utilización de métodos cualitativos - básicamente 
entrevistas e historias de vida - y la presencia muy marginal de los llamados métodos cuantitativos acompañados de análisis estadísticos.

La distribución en redes de la Sociedad europea para la investigación en educación de personas adultas, (ESREA ${ }^{1}$, por sus siglas en inglés), puede aportarnos un cuadro del estado de la investigación en EPA, al menos en Europa, en la actualidad. Se pueden enumerar las siguientes redes de investigación:

a) Sobre la ciudadanía y la democracia.

b) Sobre la formación de educadores y educadoras de personas adultas.

c) Sobre el mundo del trabajo.

d) Cuestiones relacionadas con el género.

e) Migraciones, Racismo, Xenofobia.

f) Las implicaciones de la educación de personas adultas con el desarrollo de la comunidad.

g) Sobre los recorridos de aprendizaje de las personas.

h) La utilización de historias de vida y métodos biográficos.

i) Sobre las transformaciones personales que sufren las personas en los procesos educativos, básicamente relacionadas con el Aprendizaje Transformativo.

j) La historia de la educación de personas adultas en Europa.

k) Las políticas de educación de personas adultas en Europa.

\section{Consideraciones finales}

La educación de personas adultas debería tener como foco central las personas, tratando de construir procesos participativos donde la experiencia de las mismas sea un espacio importante que permita construir situaciones de dialogo dentro y fuera del aula - en cualquiera de los espacios educativos donde la educación está siendo - y donde los procesos educativos, siguiendo los principios de la educación permanente, deberían ser reconocidos. Ese enfoque permitiría a las personas construir el conocimiento de forma colectiva ligando los saberes académicos y los vivenciales a través de metodologías como la investigación participativa que asumen la diversidad de las personas y la necesidad de integrar diferentes saberes y tendencias creando vínculos y sinergias dentro de un mismo territorio.

Otro asunto que debe destacarse es la diversidad de acciones que componen la EPA. Frente al intento reduccionista que pone el foco en una supuesta formación para ser empleable - cuando el mundo del trabajo y el de la educación constituyen dinámicas diferentes - debemos reclamar el valor de la educación por sí misma y no como un elemento aplicado a otros espacios de la vida social y/o económica (Dewey [1916] 1995). Este reconocimiento del valor de la educación en si misma nos permitirá 
reconocer y valorar la diversidad y heterogeneidad de las acciones de EPA como espacio de desarrollo y creatividad individual y colectiva.

Un último elemento a destacar tiene que ver con la necesidad de reconstruir el poder alternativo y de resistencia de la EPA para ayudar a edificar otras maneras educativas que tienen como elemento singular las conexiones entre las actuaciones educativas y las comunidades donde las personas viven. Los espacios comunitarios son el centro de la vida de las personas - tanto física como simbólicamente - y deben ser la base para la construcción de los recorridos educativos que combinen el conocimiento académico con el que las personas atesoran para co-construir un nuevo conocimiento (Orefice, 1987).

\section{Notas}

1 www.esrea.org

\section{Referencias}

Barbier, J.M. (2009). Le champ de la formation des adultes. En J.M. Barbier, E, Boureois, G. Chapelle y J.C. Ruano-Borbalan (Dirs.), Encyclopedy de la formation (pp 1- 28). Paris: PUF.

Botkin, J.W., Elmandjara M. y Malitza, M. (1979). Aprender: horizonte sin límites. Madrid: Santillana.

Coombs, Ph. (1978). La crisis mundial de la educación. Barcelona: Península.

Dale, J. y Hyslop-Margison, E. (2012). Paulo Freire: Teaching for freedom and transformation. The philosophical influences on the work of Paulo Freire. Dordrecht: Springer

Dewey, J. ([1916]1995). Democracia y Educación. Madrid: Morata.

Faure, E. et al. (1986). Aprender a ser. Madrid: Alianza.

Fejes, A. y Nylander, E. (2015). How pluralistic is the research field on adult education? Dominating bibliometrical trends, 2005- 2012. European Journal for Research on the Education and Learning of Adults, 6(2), 103-123.

Finger, M. y Asún, J.M. (2001). Adult education at the crossroads. Learning the way out. Leicester: NIACE. Fragoso, A. (2009). Desarrollo comunitario y educación. Barcelona: Diálogos

Freire, P. (1985). Pedagogía del Oprimido. Madrid: Siglo XXI.

Gelpi, E. (2004). Trabajo futuro. La formación como proyecto político. Xàtiva: Edicions del CREC.

Inglis, T. (1997). Empowerment and Emancipation. Adult Education Quarterly, 48(1), 3-18.

Jarvis, P. (1989). Sociología de la educación continua y de adultos. Barcelona: El Roure.

Käpplinger, B. (2015). Adult education research between field and rhizome - a bibliometrical analysis of conference programmes of ESREA. European Journal of Research on the Education and Learning of Adults, 6(2), 139-157.

Kurantowicz, E. (2008). Community learning. Resources, actions and reflexivity of territorial communities. In A. Fragoso; E. Kurantowicz y E. Lucio-Villegas (Eds.), Adult learning and community development (pp. 55-60). Wroclaw: University of Lower Silesia. 
Lima, L.C. y Guimarães, P. (2011). European strategies in Lifelong Learning. A critical introduction. Opladen \& Farmington Hills, Ml: Barbara Budrich Publishers.

Lucio-Villegas, E. (2009). Introduction. En E. Lucio-Villegas (Ed.), Citizenship as politics. International perspectives from adult education (pp. xiii-xv). Rotterdam: Sense Publisher.

Lucio-Villegas, E. (2016). Like a rolling stone: non-traditional spaces of adult education. European Journal for Research on the Education and Learning of Adults, 7(1), pp. 75-88.

Luria, A.S. (1987). Desarrollo histórico de los procesos cognitivos. Madrid: Akal.

Olesen, H.S. (1989). Adult education and everyday life. Roskilde: Universidad de Roskilde.

Orefice, P. (1987). Le Service comunal de Didactique Territoriale. In A. Melo (Org.), Seminario Internacional: Minoría, Áreas, Zonas desfavorecidas (pp. 337-344). Faro: Instituto Politécnico de Faro.

Rubenson, K. y Elfert, M. (2014). Changing configurations of adult education research: Exploring a fragmented map. En B. Käpplinger y S. Robak (Eds.), Changing configurations in adult education in transitional times: International perspectives in different countries (pp. 25-38). Franfurkt am Main: Peter Lang.

Schroeder, D. (2009). Informed consent: from medical research to traditional knowledge. En R. Wynberg, D. Schroeder \& R. Cennells (Eds.), Indigenous people, consent and benefit sharing (pp. 27-51). Dordrecht: Springer.

Toulmin, S. (1977). La comprensión humana l: el uso colectivo y la evolución de los conceptos. Madrid: Alianza.

UNESCO (1977). Actas de la Conferencia General. 19 reunión. Nairobi. París: UNESCO.

Ware, C. (1986). Estudio de la comunidad. Buenos Aires: Humánitas.

\section{Emilio Lucio-Villegas}

Catedrático de educación de personas adultas en la Universidad de Sevilla. Pertenece al grupo de investigación 'Estudios Sociales e Intervención Social' (ESEIS) y es investigador asociado del CeiED.

Email: elucio@us.es

ORCID: 0000-0002-8749-7296.

Correspondencia:

Emilio Lucio-Villegas

Facultad de Ciencias de la Educación

C/Pirotecnia s/n

41013 Sevilla, España

Data de Submissão: Septembro 2017

Data de Avaliação: Dezembro 2017

Data de Publicação: Dezembro 2018 